\title{
Autonomous Navigation with Sparse Reference Points
}

\author{
Isabel M. G. Lourtie \\ M. João Rendas \\ ISR/ Instituto Superior Técnico, Depart. Eng. Electr. \& Comp. \\ Av.Rovisco Pais, P-1096 Lisboa Codex Portugal
}

\begin{abstract}
This paper presents a navigation system for an autonomous underwater vehicle (AUV) that allows long range navigation by combining two distinct modes: local positioning with respect to a long baseline array of known localization (reference points) and autonomous mode in-between reference points, relying only on sonar/Doppler and depth information. In both modes, a Kalman based design approach was chosen. The performance of the proposed architecture is analyzed by Monte Carlo simulation considering a typical maneuvering operation.
\end{abstract}

\section{INTRODUCTION}

The navigation system is an essential module in any autonomous vehicle, being responsible to provide information of its localization in the operating region. In the context of underwater robotics, in the absence of a map of easily recognizable natural landmarks, the vehicle must rely on absolute positioning information furnished by another sub-system, for example reception of GPS information or localization with respect to a prespecified set of points of known position. To allow sufficient flexibility in the design of the vehicle's mission, the navigation system must be able to present a large degree of autonomy with respect to these secondary sub-systems.

In this paper we describe an autonomous navigation system that achieves long range navigation by combining two distinct modes: external positioning (local mode) with respect to long baseline arrays of known localization (reference points) and autonomous mode in-between reference points, relying on sonar/Doppler and depth information. Note that the same approach can be used if utilization of the long baseline arrays is replaced by GPS interrogation. Exclusive use of external information is in both cases limitative for general missions: for extended operating regions, implantation of a sufficiently dense network of long baseline arrays is infeasible, while GPS interrogation would require frequent displacements of the vehicle to the surface, and cannot be used, thus, for navigation close to the ocean's bottom.

This work was supported in part by the Commission of the European Communities under contracts MAST1-CT90-0059 and MAST2-CT92-0021 of the Marine Science and Technology Programmes (MAST-I and MAST-II)
With the proposed system, the vehicle is able to navigate in large areas, by periodically locking into one of the sparse reference points, where precise localization information is obtained, while admitting an increasing error level during its autonomous phase. The density of the baseline arrays is dictated by the allowable uncertainty margin resulting from periods of autonomous navigation.

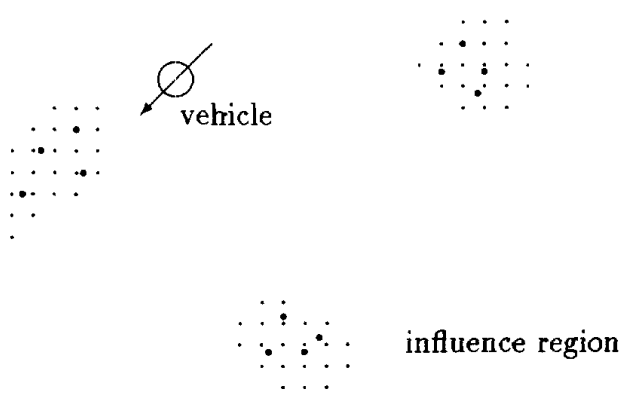

Figure 1. Partitionning of the maneuvering mission region

The covered region is divided into regions of either local or autonomous navigation [1]. The regions of local navigation are located around each baseline array, see Figure 1, corresponding to the zone where transponder based information yields reliable position estimates [2].

The areas of transponder based navigation are defined by the maximum operating range associated to each transponder. As the system is leaving the area where a particular baseline array is located, the number of range measurements it is able to receive will gradually decrease to zero, entering, in this way, the autonomous navigation mode. On the contrary, when it approaches an area where transponders are located, it receives an increasing number of distance measurements, switching from autonomous to local navigation mode.

The paper is organized in the following way. In section II we briefly describe the navigation system under both local and autonomous modes. Simulation results of the navigation system are presented for a typical maneuvering situation in section III. Finally, section IV presents the conclusions of the paper. 


\section{NAVIGATION SYSTEM}

A. Introduction

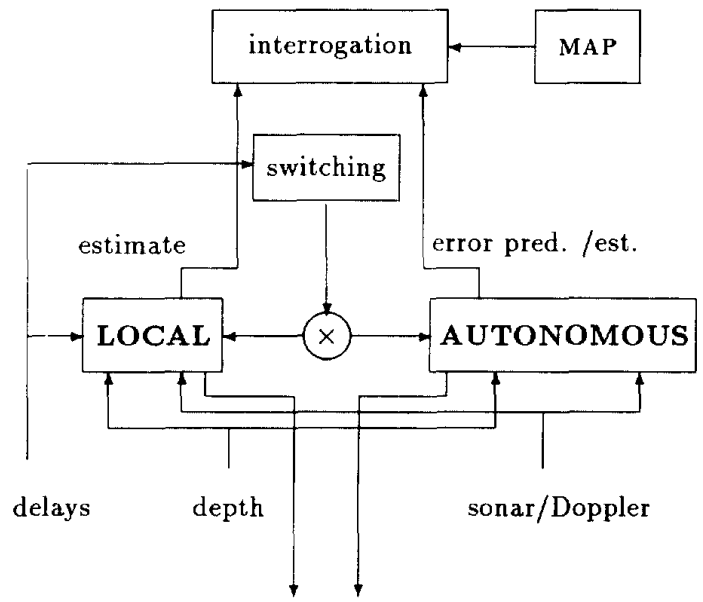

position and velocity estimates

Figure 2. Functional block diagram.

The navigation system, see Figure 2 , is, at any time instant, in either autonomous or local mode [1]. The interrogation block uses the estimated vehicle's position and the error prediction to determine its proximity to one of the baseline arrays, deciding on the need to send an interrogation signal, that is, the possibility of operating in local mode.

A switching module determines, based on the number of transponder answers, the convenient navigation mode (local or autonomous). While in the local mode of navigation the vehicle produces position estimates from knowledge of locally gathered information (depth, sonar/Doppler) as well as the round trip travel times to the elements of the long baseline array, in the autonomous mode the vehicle uses only depth and sonar/ Doppler information, as sown in Figure 2.

For both local and autonomous modules, a Kalman filtering model based design approach was used. Since most of the time the expected vehicle's acceleration is close to zero, becoming non-zero only during maneuvering situations, we implemented a variable dimension Kalman filter [3]. When the vehicle's movernent is uniform, the Kalman filter assumes the acceleration is zero and estimates just the position and the linear velocity (quiescent model). Each time a maneuver is detected, the Kalman filter switches to a larger order (maneuvering model) extending its state vector to include the vehicle's accelerations.

This paper is mainly concerned with the local and autonomous modules of the navigation system, the design of the interrogation block being left for future research.

\section{B. Autonomous Navigation}

The autonomous navigation system, see Figure 3 , relies on sonar/Doppler and, when available, depth information. It consists on a Kalman filter that propagates the vehicle's dynamics according to both the measured values of the linear velocity along each coordinate, and the input of the depth sensor [1].

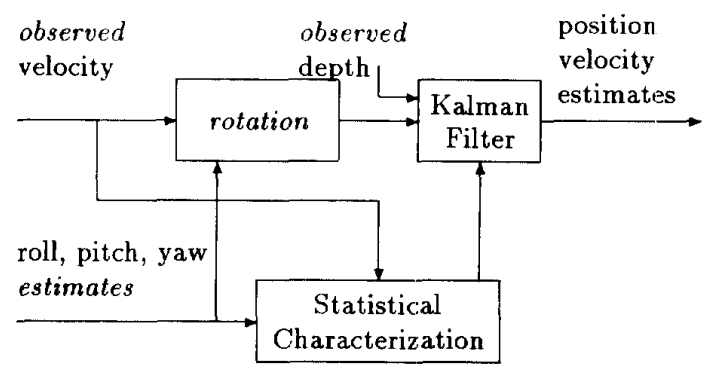

Figure 3. Autonomous navigation system.

The velocity measurements provided by the sonar/ Doppler system are reported to the vehicle's coordinate system (body frame). Since the navigation system operates in a global inertial frame, a rotation transformation must be applied, depending on the values of the roll, pitch and yaw angles, that relate the two coordinate systems. This transformation is performed by the block rotation in Figure 3.

In practice, angles roll, pitch and yaw are not accurately known but measured (or estimated) on-board the vehicle. Consequently, when transforming the velocity measurements from the body to the inertial frame, an aditional error is introduced in each component of the velocity.

Due to the non-linear dependence on the roll, pitch and yaw angles of the rotation transformation matrix, the input noise to the Kalman filter is not stationary. To minimize the effects of this non-stationarity, one must assure that an accurate statistical characterization of the observations at the filter input is available, at each time instant.

The statistical characterization of the velocity (with respect to the inertial frame) measurement vector, which represents part of the Kalman filter observations in both autonomous and local modes of navigation, is performed on-line, being accomplished by taking a first order approximation to the expressions that describe the rotation transformation that relates the velocity vectors on both (inertial and body) frames. The measurement error covariance matrix is computed assuming that velocity and angular measurements are mutually independent Gaussian processes. 


\section{Local Navigation}

The local navigation mode assumes, in addition to odometry data (depth and sonar/Doppler measurements), the existence of an hydrophone/ projector unit located on-board the vehicle, and of an array of four subsea transponders located near the sea bottom, at ranges that allow transponder interrogation by the vehicle. The twoway range between the vehicle and each transponder unit is computed based on the round-trip transmission time.

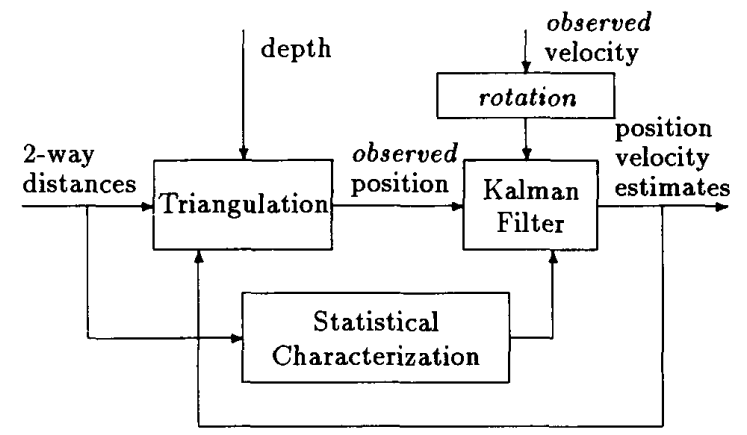

Figure 4. Local Navigation System.

Figure 4 represents a block diagram of the local navigation system [2]. The triangulation block performs the geometric transformation between the measured ranges and the vehicle's position, while the Kalman filtering

block carries out the temporal filtering of the observed position and velocity.

The triangulation routine takes into account the possibility of misfunction (no measurement) of one or more transponder units, and/ or the presence of outliers in the range measurements, that is, it computes a measurement of the vehicle's position provided that at least one range measurement is available. This is particularly important when the vehicle is either leaving or approaching an area covered by one of the baseline arrays.

For both autonomous and local modes of navigation, the Kalman filter structure is essentially the same, with a convenient interpretation of the observation vector. However, while in the autonomous mode a periodic Kalman filter is implemented, in the local mode a non-periodic one is considered due to the non-constant measurement rate of the delays.

As for the velocity measurement vector, the on-line statistical characterization of the highly non-homogeneous errors in the computation of the vehicle's linear position [2] is required. To compute its covariance matrix we assume that (i) the Kalman filter position estimate and the depth measurement are mutually independent zero mean Gaussian processes, and (ii) the range measurements are mutually independent uniformly distributed random variables.
The local navigation system is presented and described in [2], including the analysis and discussion of the nonhomogeneous character of the position measurement error.

\section{SIMULATIONS}

In this section we present simulations of the navigation system for a typical vehicle trajectory. The case-studies considered were designed in order to analyze and compare the behavior of the navigation system as a function of (i) the existence of reference points, (ii) the trajectory depth, and (iii) the availability of depth measurements.

\section{A. Scenario}

In the simulated scenario, two base-line arrays of four transponder units are available. Each base-line array defines a square in the horizontal $(x, y)$ plane with $70 \mathrm{~m}$ sides, which are centered around positions $(35,35) \mathrm{m}$ and $(1135,585) \mathrm{m}$. All transponder units are located in the sea bottom, at a depth of $100 \mathrm{~m}$, their maximum range of operation being $150 \mathrm{~m}$.

\section{B. Trajectory}

The simulated trajectories are linear, being described in the horizontal plane at depth $z_{0}$. In the $(x, y)$ plane, see Figure 5, the two trajectories are identical just differing in the $y$ component of the vehicle's initial position.

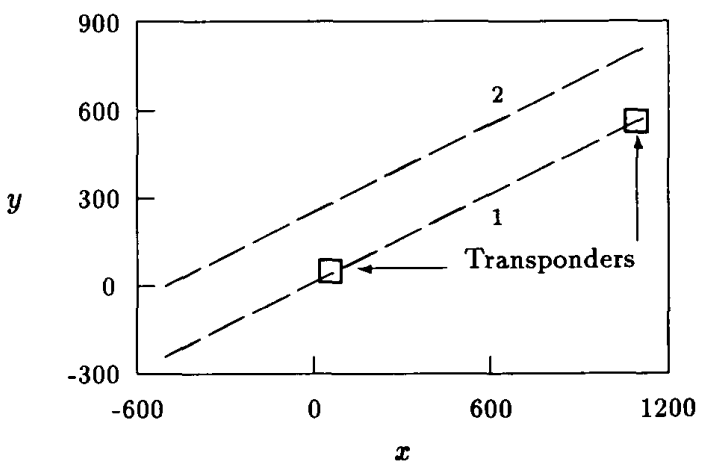

Figure 5. Vehicle trajectories and base-line arrays localization.

In the first trajectory (1), the vehicle starts its motion at point $\left(-500,-245, z_{0}\right)$, and navigates towards the two arrays in stationary motion. In this way, we simulate both modes of navigation, that is, in-between (autonomous) and inside (local) reference areas. In order to get insight on the navigation system behavior when operating in the autonomous mode, we considered a second trajectory (2), defined by the vehicle's initial position $\left(-500,0, z_{0}\right)$, for which the vehicle never approaches the two baseline arrays. 
For both cases, during the first and last $80 \mathrm{~s}$ the vehicle's motion is uniformly accelerated (positive acceleration of $0.02 \mathrm{~ms}^{-2}$ and $0.01 \mathrm{~ms}^{-2}$ in the $x$ and $y$ directions, respectively, for the first segment, and symmetric values for the last one). In-between, an uniform motion is imposed (positive velocity of $1.6 \mathrm{~ms}^{-1}$ and $0.8 \mathrm{~ms}^{-1}$ in $x$ and $y$ components, respectively). The vehicle completes its motion at time $T=1100 \mathrm{~s}$.

\section{Measurement Errors}

The round-trip range measurement errors are modeled as symmetric mutually independent uniform random variables in the interval $\left[\begin{array}{lll}-0.15 & 0.15\end{array}\right] \mathrm{m}$. Both depth and velocity components measurement errors are assumed to be mutually independent zero mean Gaussian random variables, with standard deviations $0.2 \mathrm{~m}$ and $0.05 \mathrm{~ms}^{-1}$, respectively.

\section{Case-Studies}

'Table I describes the case studies considered, the different columns defining the actual conditions: Traj represents the vehicle's trajectory, $z_{0}$ denotes the vehicle's initial depth, and DS is a yes/ no variable indicating the pres. ence or absence of depth measurements.

Table I

Case-Studies

\begin{tabular}{|c||c||c|c|c|}
\hline Case study & Fig. & Traj. & $z_{0}(\mathrm{~m})$ & DS \\
\hline \hline A & 6 & 2 & 60 & no \\
\hline B & 7 & 2 & 60 & yes \\
\hline C & 8 & 1 & 60 & no \\
\hline D & 9 & 1 & 60 & yes \\
\hline E & 10 & 1 & 90 & no \\
\hline F & 11 & 1 & 90 & yes \\
\hline
\end{tabular}

Monte Carlo experiments (50 runs) were conducted, the position mean square error along each coordinate being computed.

Figures 6 (case A) and 7 (case B) show the navigation system behavior when operating always in the autonomous mode. As expected, when depth measurements are available (case B) the level error in the $z$ component is very small remaining constant independently of the time duration of the autonomous navigation. On the contrary, when no depth measurements are provided (case A) the mean square error in all directions increases linearly with time. Notice, however, that if no filtering was performed, and the vehicle's position computed directly by integration of the velocity measurements, the slope of the position mean square error for this case study is $0.0025 \mathrm{~m}^{2} \mathrm{~s}^{-1}$, which is about one order of magnitude larger than the result obtained with the Kalman filter.

The effect of the presence of reference areas along the vehicle's trajectory, leading to regions of transponder based navigation (local mode), is shown in Figures 8 to 11 (cases $\mathrm{C}$ to $\mathrm{F}$ ).
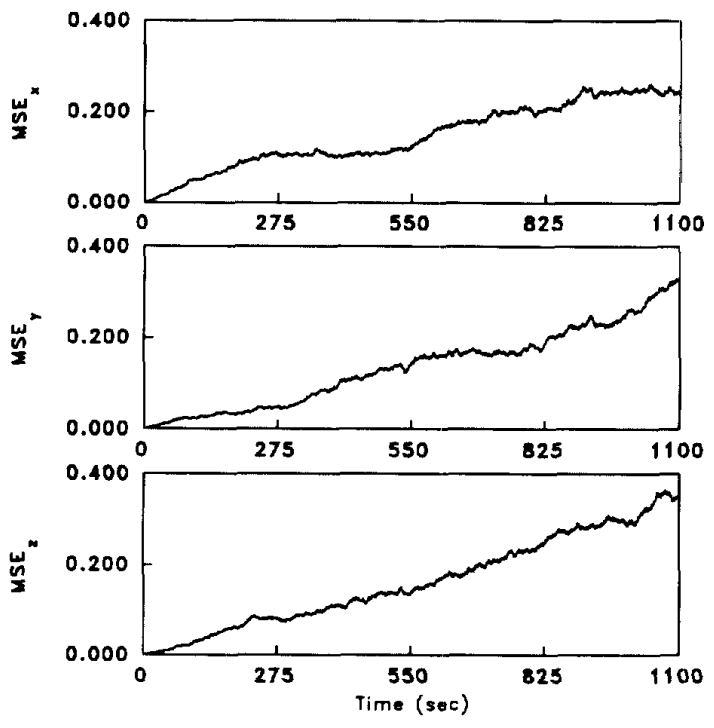

Figure 6. Position mean square error - case $\mathrm{A}$

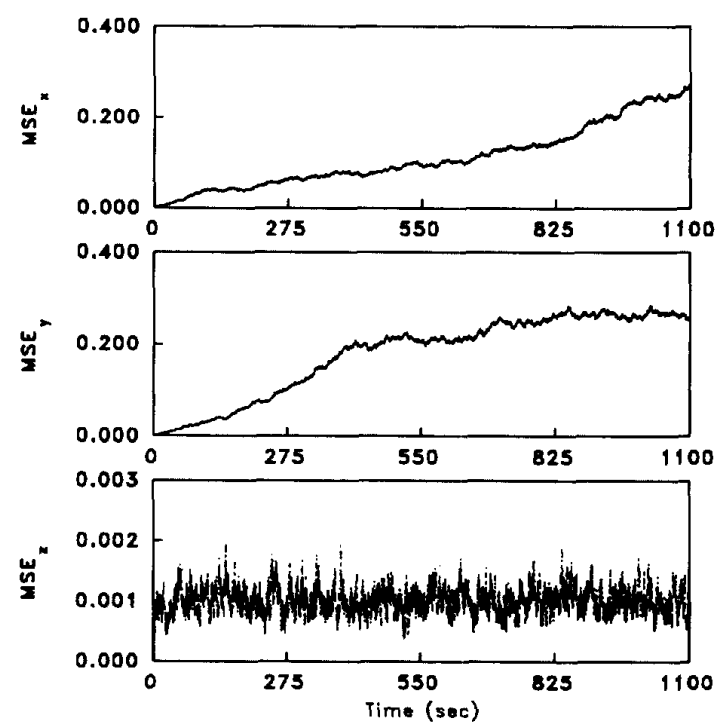

Figure 7. Position mean square error - case B

For cases $C$ and D, respectively Figures 8 and 9 , the vehicle's trajectory is within the influence region of the first base-line array for $271 \mathrm{~s} \leq t \leq 483 \mathrm{~s}$. However, just 

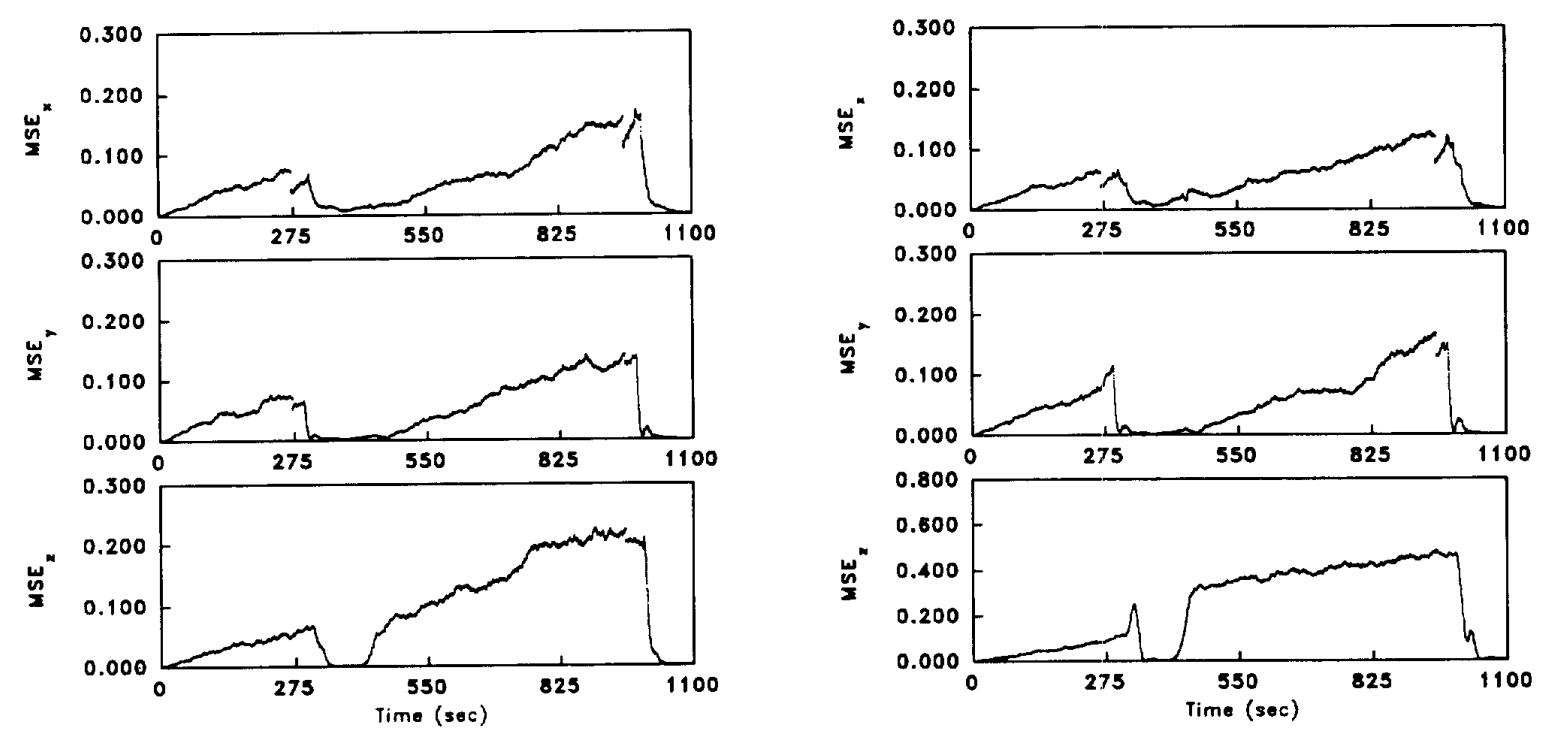

Figure 8. Position mean square error - case C
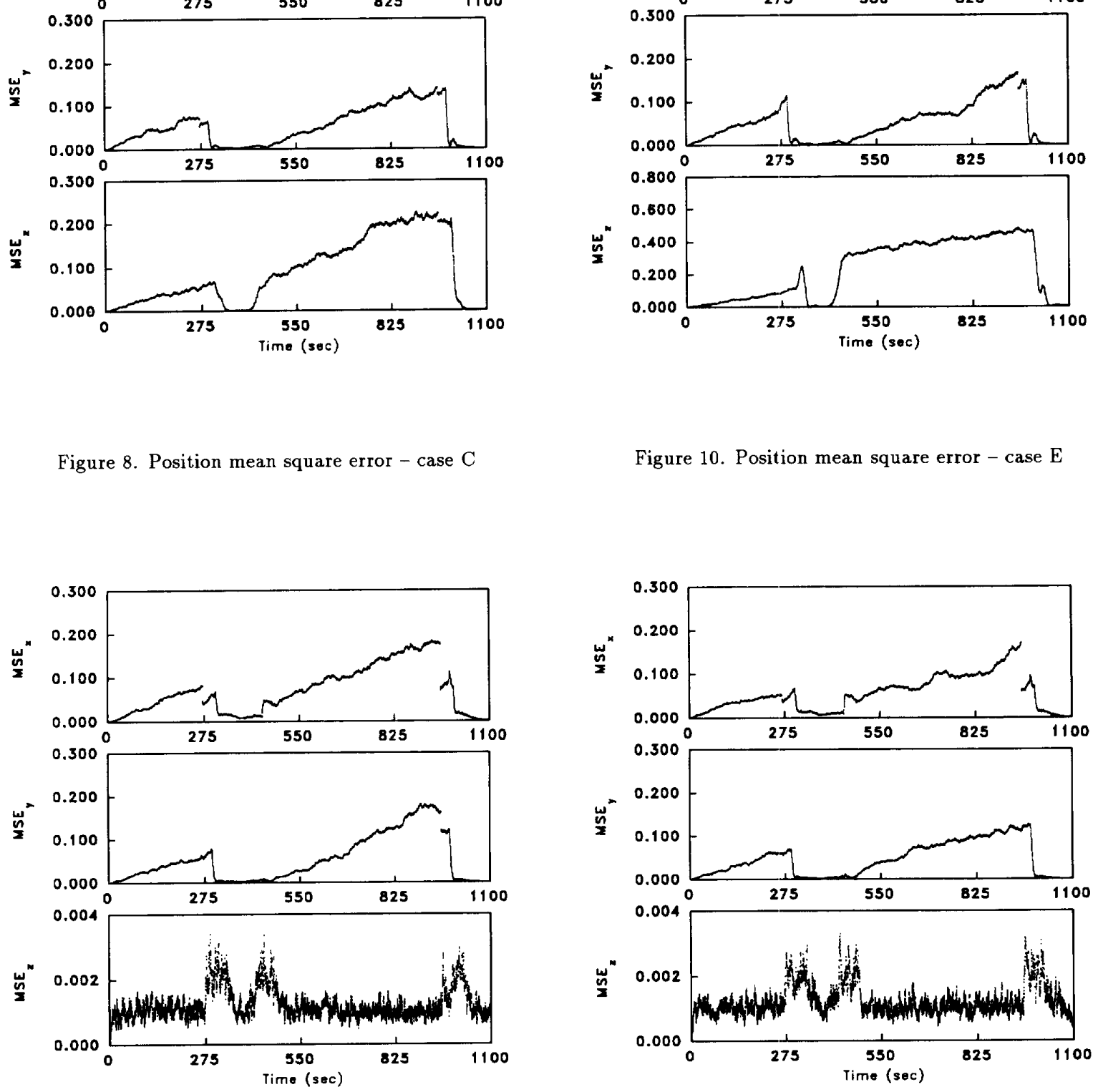

Figure 9. Position mean square error - case D

Figure 11. Position mean square error - case F 
for $324 \mathrm{~s} \leq t \leq 432 \mathrm{~s}$ the four transponder units are active, corresponding to the smaller error values obtained. In the transition between autonomous and local modes, a transient effect in the error is observed. A fast decrease in the error is obtained only after the third transponder becomes active. As expected, outside the influence region of the transponders, that is, under autonomous navigation, the error increases linearly.

A similar qualitative behavior is obtained for cases $\mathrm{E}$ and $F$, respectively Figures 10 and 11 . Notice that, this trajectory being deeper, that is, closer to the transponder units, the influence region of the base-line array is now slightly larger.

It was shown in [2] that, under transponder based navigation, the $z$ component of the mean square error increases for trajectories approaching the base-line array. This fact explains the larger value observed for the $z$ component of the mean square error in Figure 10 when compared to the one shown in Figure 8. Notice that, as expected, under the autonomous mode of navigation the slope of the time evolution of the mean square error remains independent of the vehicle's depth.

On the contrary, when depth measurements are provided, Figures 9 and 11 (cases D and F), the smaller error is observed along the $z$ coordinate, being independent of the vehicle's depth. This is a consequence of the good observability conditions due to the additional depth measurement provided by the dedicated sensor. The peaks observed in the $z$ component of the mean square error correspond to regions where just one or two transponders are active.

\section{CONCLUSIONS}

In this paper we presented a navigation system that allows extended range operation, suitable for supporting missions where several small dispersed areas must be visited, and inside which accurate positioning of the autonomous vehicle is necessary. The system automatically switches from a transponder-based operating mode to an autonomous mode when no transponder measurements are available.

In this last mode, it relies on on-board odometry measurements (depth sensor and sonar/Doppler velocity information), to drive a Kalman filter that yields position and velocity estimates. During periods of autonomous navigation the uncertainty regarding position increases linearly, due to the errors associated to the velocity measurements.

Although the slope of the increased mean square during autonomous navigation depends on the accuracy of the sonar/Doppler velocity measurements, as analyzed in [1], we observed in this paper that the use of a Kalman filter with appropriate statistical characterization of its inputs, leads to a drastic reduction of the error when compared to the one that results from just integrating the velocity measurement data.

Therefore, the precision of the velocity measurement mechanism dictates the maximum separation of reference points. However, while the use of a good sonar/Doppler system along with appropriate filtering allow base-line arrays to be spread in a sparse way, a noisy velocity measurement system imposes strict limits on the periods of autonomous navigation.

Whenever the system is approaching a base-line array, it gradually incorporates available measurements of distance, being in this way able to significantly improve the precision of the position estimates. The error behavior during transponder-based periods has been analyzed and discussed in [2], with respect to transponder placement and to the availability or not of a dedicated depth sensor. In [2], we concluded that, for local navigation, the availability of depth measurements leads to both homogeneity and a drastic reduction on the vehicle's depth positioning error, being of paramount importance to guarantee system robustness, in particular, under missing transponder measurements.

For autonomous navigation, we show in this paper the importance of having a dedicated depth sensor on achieving a reduced and bounded error in the vehicle's depth.

Future work will address the question of establishing simple analytical approximations to the error evolution during autonomous navigation, to be used for determining the layout of the base-line arrays, and to derive the constraints on path planning once a layout has been decided upon.

\section{ACKNOWLEGEMENTS}

Part of the work of the second author was done while on leave at Laboratoire I9S, CNRS URA 1376, Université de Nice Sophia Antipolis, France.

\section{REFERENCES}

[1] M. João Rendas, Isabel M. G. Lourtie, "Hybrid Navigation System for Autonomous Underwater Vehicles Long Range Operation," Proceedings of the Symposizm on Autonomous Underwater Vehicle Technology, Cambridge, Massachusetts, USA, July 1994.

[2] Isabel M. G. Lourtie, M. João Rendas, "A Transponder Based Navigation System for Autonomous Underwater Vehicles," Proceedings of the Workshop on Autonomous Underwater Vehicles, Porto, Portugal, September 1993.

[3] Yaakov Bar-Shalom, Thomas E. Fortmann, Tracking and Data Association, Academic Press Inc., 1988. 